\title{
Risk Management of Neuroanesthesia in Traumatic Brain Injury with Multiple Facial Fractures and Subcutaneous Emphysema in terms of Ethical and Medicolegal Aspects
}

\author{
$\underline{\text { Kulsum}}^{1}$, Taufik Suryadi ${ }^{2}$ \\ ${ }^{1}$ Department of Anesthesiology and Intensive Therapy, Faculty of Medicine, Universitas Syiah Kuala, \\ Banda Aceh, Indonesia \\ 2Department of Forensic Medicine and Medicolegal, Faculty of Medicine, Universitas Syiah Kuala, \\ Banda Aceh, Indonesia \\ Email: kulsumanest@unsyiah.ac.id
}

\begin{abstract}
:
Every medical practice including neuroanesthesia always contains risks, so risk management is needed. Neuroanesthesia in cases of traumatic brain injury (TBI) with multiple facial fractures and subcutaneous emphysema is one of the risky prosedure that must be done very carefully and professionally. A case reported by a man, 53 years old, weight $80 \mathrm{~kg}$, decreased consciousness due to head trauma and facial trauma and the presence of widespread subcutaneous emphysema. Performed risk management in the form of airway management, installation of water seal drainage and perioperative neuroanesthesia aimed at reducing intracranial pressure and maintaining adequate cerebral perfusion pressure. Medical decision making in this case does not only give attention to medical aspects but also must give attention to aspects of clinical ethics and medico-legal. Criteria for TBI patients with multiple facial fractures and subcutaneous emphysema for neuroanesthesia based on ethical and medicolegal considerations can be carried out based on clinical ethics theory and neuroanesthesia risk management so as to produce good patient outcomes.
\end{abstract}

Keywords:

clinical ethics; neuroanasthesia; risk management

\section{Introduction}

Anesthesiology is a medical science that is always oriented to patient safety. However, complications can occur that can not be prevented, prompt and appropriate handling of the potential risks detected is needed (Cabrini \& Levati, 2009). Because of the complexity of anesthesia and surgery, risk management is very important to reduce the morbidity and mortality associated with anesthesia, although the anesthetic prosedure is not alone in determining the success of surgery because it is also influenced by the patient's health status and the complexity of the operation (Cevik \& Yuce, 2018). Every medical prosedure including neuroanesthesia always contains risks so that risk management is needed (Sampurna, 2004). Neuroanesthesia in cases of traumatic brain injury (TBI) with multiple facial fractures complicating the complications of subcutaneous emphysema is one of the risky measures so it must be done very carefully and professionally (Lienhart et al., 2006).

TBI is a head trauma that causes impaired brain function with clinical symptoms such as confusion, decreased consciousness, seizures, coma, and motor and sensory disorders as well as neurological deficits. According to Yue (2020) the cognitions and thoughts of a human brain are accomplished by the neurons to transmit informations in brain. If we want to know the thoughts of a brain, we must to contact the neural network of the 
brain. In mild head trauma, symptoms that often arise are behavioral changes and neuropsychological changes. The bleeding that occurs in patients with head trauma is a clue for medical personnel to find out the location of head trauma. Bleeding due to intracranial trauma usually occurs in the epidural space, subdural space, subarachnoid space, brain tissue (intra cerebral hemorrhage) and cerebral ventricles (Aghakhani, et al., 2014; Farrell \& Bendo, 2018). TBI patients almost always undergo general anesthesia with the aim of surgery, radiological investigations, or to improve mechanical ventilation. TBI can result in head bone fractures, brain bruising, and injury to blood vessels and brain parenchyma which can cause intracranial bleeding and increased intracranial pressure (ICP). This process continues until inflammation and brain edema that will worsen the increase in ICP and ultimately can reduce the pressure of brain perfusion (PBP) (Syah et al., 2017).

With the diagnosis of TBI with multiple facial fracture complications and complicated complications of subcutaneous emphysema, the patient's disease prognosis can be predicted. Prognosis in this patient dubia et malem (leads to poor). As it has been understood that the effects of TBI in general can be recognized clinically consisting of 2 types, namely the primary effect of direct cranioserebral injury that occurs during trauma and secondary effects of cranioserebral injury due to primary damage complications such as cerebral edema, cerebral blood damage, necrosis tissue, hyperthermia, and others (Lalenoh et al., 2012). Medical decision making in this case does not only give attention to medical aspects but also must give attention to aspects of clinical and medical ethics. Criteria for TBI patients with multiple facial fractures and subcutaneous emphysema who will be given neuroanesthesia based on ethical considerations can be carried out based on clinical ethical theory and anesthetic risk management (Jonsen et al., 2010).

\section{Case Report}

A man, 53 years old, was referred by the regional hospital with a history of decreased awareness due to traffic accidents. According to family information, after the incident the patient was unconscious, vomiting was found, bleeding from the nose, mouth and ears. Also found fractures in the facial bones. Shortness of breath found and the whole body looks even more swollen when in the hospital area treatment. Physical examination and preoperative management in the ER found the patient in a hemodynamically unstable condition, respiratory rate 30 times / minute and increased work of breathing, asymmetrical chest wall movement and lesions on the left chest, on palpation there was crepitation on the face, neck, chest, abdomen and scrotum. Physical examination of the head found a fracture of the temporoparital os sinistra, os frontal dextra and os arcus zygomaticus sinistra. On physical examination found problems with swollen neck, plica vocalis edema, fractures in the zygoma, swollen hands and thighs, swollen scrotum, crepitation of the neck and chest. The patient was diagnosed with TBI with multiple facial fracture complications and subcutaneous emphysema. This situation disturbs the airway, breathing and circulation, so that the risk that occurs in the state of facial fractures and subcutaneous emphysema causes difficult intubation. So that risk management steps are needed to reduce morbidity and mortality and also discuss the ethical and medicolegal aspects of risk management.

\section{Discussion}

Risk management in neuroanesthesia includes preventive measures and management evaluations that have been carried out to reduce morbidity and mortality (Cabrini \& Levati, 2009). The American Society of Anesthesiologists (ASA) has made a risk classification in the field of anesthesia that has been used extensively (Kumar \& Kumar, 2015; Cevik \& Yuce, 
2018). Risk management is an effort that tends to be proactive and can also be an evaluation of previous experience to be applied in an effort to reduce or prevent similar problems in the future (Sampurna, 2004). There are four steps of risk management in the neuroanesthesia: (1) detection of problems (2) assessing the problem (3) resolution of the problem, and (4) verification (Cabrini \& Levati, 2009). These patients are categorized in ASA level 3-4 status with moderate to high risk. Risk management in this case includes the steps mentioned above.

The first step is detection of problems, in this case patients with clinical asymmetrical chest movements supported by chest x-ray examination found a left posterior fracture, which causes an increased risk for pneumothorax which progresses to subcutaneous emphysema. Subcutaneous emphysema is a condition where there is air between the tissues under the skin. Many things can cause subcutaneous emphysema including sharp trauma, pneumothorax barotrauma, infection, malignancy, or complications from several surgical procedures. The most typical symptom that appears is swelling around the neck accompanied by chest pain. Other symptoms include sore throat, difficulty swallowing, pain in the neck area, difficulty breathing, wheezing, and distension. Complications of subcutaneous emphysema can cause disruption of full lung expansion and can be followed by increased airway pressure, severe respiratory acidosis, ventilation failure, pacemaker malfunction, and tension phenomena (Aghajanzadeh et al., 2015).

The second step is assessing the problem, which is difficult for intubated patients because of the subcutaneous emphysema which extends mainly in the cervical area to cause epiglottis and paratracheal swelling to completely cover the anatomy and landmarks for intubation. The management of subcutaneous emphysema is carried out simultaneously by overcoming the cause. The main thing done is a subcutaneous incision using a needle, drain and cervical mediastinostomy (Aghajanzadeh et al., 2015). Multiple Fracture is a condition where there is loss of continuity of bone tissue in more than one line caused by external pressure characterized by pain, swelling, deformity and impaired function in the fracture area (Barak et al., 2015). TBI cases accompanied by subcutaneous emphysema and multiple fractures on the face can cause a difficult problem of intubation. So that airway management is needed, and appropriate interventions are needed to reduce morbidity and mortality.

The third step, resolution of problem, is the management of the airway in the form of tracheal intubation (Curry et al., 2011). The fourth step is verification, the installation of intubation puts a positive pressure on the patient that causes the expansion of existing subcutis emphysema. Therefore, installation of a Water Seal Drainage (WSD) before intubation is needed to expel trapped air in the pleural cavity so that it does not worsen the condition of subcutaneous emphysema (Olmstead, 2018). Perioperative management of neuroanesthesia starting from the emergency room, in the operating room and in the Intensive care Unit (ICU) also influences the outcome of the patient's postoperative condition (Barak et al., 2015).

Basically, risk management is an ongoing cyclic process, generally consisting of four stages namely risk awareness, risk prevention, risk reduction, and risk transfer (Kohn et al, 2000). However, clinical risk management in neuroanesthesia is carried out in five stages namely risk awareness, risk identification, risk assessment, risk management and re-evaluation (Bould et al., 2006). The medical risks associated with anesthesia are often used as benchmarks for improving quality in hospitals (Haller et al., 2011).

To reduce the risk of post-surgery, extubation of this patient is not performed due to severe cerebral edema and disruption of ventilation, as in general neurosurgical surgery of 
patients is awakened from anesthesia as soon as possible, so that neurological status can be evaluated immediately as a result of surgery. It is also in accordance with the procedure that in patients with severe head injury there is cerebral edema or potentially having cerebral edema, extubation is not done immediately so there is the term slow weaning and delayed extubation. Late emergence can be performed on conditions: poor preoperative level of consciousness, risk of edema or aggravation of edema such as long surgeries, heavy bleeding, near vital areas, extensive operations, preoperative difficult airway management (Butterworth et al., 2013; Farrell \& Bendo, 2018).

In this patient risk management is performed on neuroanesthesia which is focused on correction of primary brain injury and avoiding secondary brain injury, early resuscitation, hemodynamic stabilization, and emergency surgery for evacuation of bleeding by providing adequate surgical conditions (slack brain), reducing ICP by decreasing cerebral blood Flow $(\mathrm{CBF})$, reduces Cerebral Metabolic Oxygen Rate (CMRO2), maintains Cerebral Perfussion Pressure (CPP), and optimizes brain Oxygen Delivery (DO2), protects nerve tissue from ischemia and injury (Brain Protection). (Syah et al., 2017; Basuki et al., 2015; Bisri, 2012).

By using the ABCDE Neuro Anesthesia principle: A) airway, freeing airway all the time, B) breathing, controlling ventilation to get adequate oxygenation and normocapnea, C) circulation, avoiding excessive blood pressure increase or decrease, avoiding mechanical factors that increase pressure cerebral veins, maintain conditions of normotension, normoglycemia, isoosmolar during anesthesia, D) drugs, avoid drugs and anesthesia techniques that can increase ICP, and give drugs that have a protective effect of the brain, E) environment, temperature control with a core temperature target of $35 \mathrm{oC}$ at operating room. Patients brought to the operating room are already in control of ventilation because they are intubated in the emergency department, and during the diagnostic measures are still carried out ventilation control with relaxants (Shah et al., 2017; Basuki et al., 2015; Bisri, 2012).

In addition to risk management, it is also necessary to discuss ethical and medicolegal aspects in making medical decisions for patients. Determination of medical indications in TBI patients can be done using the principle of beneficence and nonmaleficence. The Beneficence Principle means that therapy must provide medical benefits, while non-maleficence means don't harm the patient both medically and financially (Jonsen et al., 2010). TBI is a big problem in the medical world because of the high mortality and morbidity that are generally caused by traffic accidents (Lalenoh et al., 2012). There are three goals for anesthesiologists besides facilitating surgery, they are (1) controlling intracranial pressure and brain volume, (2) protecting nerve tissue from ischemia and injury, (3) reducing bleeding (Bisri, 2012). Successful management of primary and secondary brain injuries can affect the quality of life after anesthesia and surgery.

Measurement of quality of life is determined using the principles of beneficence, nonmaleficence and autonomy. Quality of life is a form of satisfaction, value statement, life experience in all aspects for better or worse (Jonsen et al., 2010). Determination of the quality of life of patients after anesthesia needs to be considered because the condition of preanesthesia patients is already bad. In this condition, ethical considerations can be carried out with the prima facie approach by establishing the interests of patients or with the minus mallum approach by choosing more minimal harms or with the double effect principle because the only way to take is surgery (Beauchamp \& Childress, 2013).

The patient and / or family's request also needs to be served using the Autonomy principle. Autonomy means that every medical action must be approved by a competent 
patient (or his immediate family, in the event that the patient cannot give his consent). Ethical and medicolegal standards for decision making are based on decisions made for patients. Although clinical ethics is more focused on medical indications, patient demand and quality of life, medical decisions are not only made by doctors and patients but must also consider other aspects such as socio-cultural, religious, religious and financial. Contextual features are obtained by using the principles of justice and fairness (Jonsen et al., 2010).

\section{Conclusion}

According to Suryadi (2020) ethical problems when patients leave ICU can also arise. Based on the research of Oerlemans et al, found ethical problems in the form of the end of the indicative period of treatment given, differences of opinion about the cessation of management in the ICU, the patient returned to stability after the termination of life assistance therapy and the patient was stable during administration and treatment at the ICU. Medical decision making coupled with risk management as well as ethical and medicolegal decisions is highly recommended. In this patient, risk management has been carried out as well as ethical and medicolegal considerations that produce the best decisions for patients. With continuous risk management, it can reduce or prevent similar problems in the future. Determination of quality of life of patients after anesthesia needs to be considered because the condition of pre-anesthesia patients is already bad. In this condition, ethical considerations can be made using the prima facie approach, the minus mallum principle and the double effect principle.

\section{References}

Aghakhani K, Heidari M, Yousefinejad V, Okazi A. (2014). Frequency of intracranial injuries in cadavers with head trauma with and without scalp injuries in Tehran. J Forensic Leg Med. 28: 36-38.

Aghajanzadeh M, Dehnadi A, Ebrahimi H, et al. (2015). Classification and Management of Subcutaneous Emphysema: a 10-Year Experience. 77 (December): 2-6.

Barak M, Bahouth H, Leiser Y, et al. (2015). Airway Management of the Patient with Maxillofacial Trauma: Review of the Literature and Suggested Clinical Approach.

Basuki WS, Suryono B, Saleh SC. 2015. Perioperative Management of Severe Traumatic Head Injury with Cushing Signs. Indonesian Neuroanesthesia Journal. 4 (1): 34-42.

Beauchamp, T.L., Childress, J.F. (2013). Principles of biomedical ethics (7th ed). New York: Oxford University Press.

Bisri T. (2012). Management of Neuroanesthesia and Critical Care Traumatic Brain Injury. Publisher of the Faculty of Medicine, Padjadjaran University. Bandung, 83-124.

Bould. M.D, Hunter. D, and Haxby. E. J, (2006). Clinical risk management in anesthesia, Continuing education in anesthesia, critical care and pain. 6(6): 240-242.

Butterworth JF, Mackey D, Wasnick JD. (2013). in Morgan and Mikhail's Clinical Anesthesiology. Fifth Edition. New York: McGraw-HilL.

Cabrini. L, and Levati. A. (2009). Risk management in anesthesia. Minerva anesthesiologica vol. 75, no.11: pp.638-643.

Cevik. B, and Yuce. Y. (2018). Risk assessment in surgical patients: American Society of Anesthesiologists (ASA) Classification vs. Intraoperative Therapeutic and Diagnostic Interventions (I-ITS), American Journal of Clinical Medicine Research, vol. 6, no. 1, pp.15-19.

Curry P, Viernes D, Sharma D. (2011).Perioperative Management of Traumatic Brain Injury. 1 (1): 27-35. 
Farrell D and Bendo AA. (2018). Perioperative Management of Severe Traumatic Brain Injury: What Is New?: 279-89.

Haller. G, Laroche. T, and Clergue. F. (2011). Morbidity in anesthesia: today and tomorrow, Best practices \& research clinical anesthesiology, vol.25, pp.123-132.

Jonsen AR, Siegler M, Winslade WJ. (2010). Clinical ethics: a practical approach to ethical decisions in clinical medicine. 7th edn. Philadelphia, USA: McGraw-Hill Medical Publishing Division: 9-225.

Kohn LT, Corrigan JM and Donaldson MS. (2000). To err is human, building a safer health system. Washington DC: National Academy Press, pp.58-60.

Kumar. R, and Kumar. A, (2015). Anaesthetic mortality: a clinical and medico-legal scenario, International Journal of Science and research, vol.4, issue 7, pp. 316-320.

Lalenoh DC, Sudjito NH, Suryono B. (2012). Treatment of anesthesia in traumatic brain injury. Indonesian Neuroanesthesia Journal. 1 (2): 120-132.

Lienhart. A, Auroy. Y, Pequignot. F, Benhamou. D, Warszawski. J, Bovet. M, et al. (2006). Survey of anesthesia-related mortality in France, Anesthesiology, vol.105, no.6, pp.10871097.

Olmstead D, Gelfand G, Anderson I, Kortbeek JB. (2018). A Case Report of Acute Airway Compromise due to Subcutaneous Emphysema: 10.

Sampurna. B. (2004). The medicolegal aspects of present medical services and their relation to clinical risk management. Unpublished.

Suryadi, T., and Kulsum (2020). Identification of Ethical and Medicolegal Problems in the Care of Patients in the Intensive Care Unit. Britain International of Exact Sciences (BIoEx) Journal Vol. 2 (2): 547-555.

Syah BIA, Suarjaya IPP, Rahardjo S, Saleh SC. (2017). Anesthesia Management in Severe Head Injury Patients from Acute Epidural Hematoma with Pregnancy. Indonesian Neuroanesthesia Journal. 6 (3): 169-177.

Yue, Z. (2020). We are Telling the World Everything the Cerebral Cortex Memorises. Britain International of Exact Sciences (BIoEx) Journal Vol. 2 (2): 486-488. 